\title{
Smart Real-Time Recommendation of Mobile Services
}

\author{
IVAN GANCHEV \\ Department of Computer Systems, \\ University of Plovdiv "Paisii Hilendarski", \\ 24 Tsar Assen St., Plovdiv 4000, \\ BULGARIA. \\ $\&$ \\ Institute of Mathematics and Informatics, \\ Bulgarian Academy of Sciences, \\ Akad. G. Bonchev St., Block 8, Sofia 1113, BULGARIA. \\ https://orcid.org/0000-0003-0535-7087 \\ $\&$ \\ Telecommunications Research Centre (TRC), \\ University of Limerick, \\ Plassey, National Technological Park, Co. Limerick, \\ IRELAND. \\ ZHANLIN JI \\ College of Artificial Intelligence, \\ North China University of Science and Technology, \\ Caofeidian, Tangshan City, \\ CHINA. \\ https://orcid.org/0000-0003-3527-3773
}

\begin{abstract}
In this paper, a new vision is presented for highly personalized, customized, and contextualized realtime recommendation of services to mobile users (consumers) by considering the current consumer-, network-, and service context. A smart service recommendation system is elaborated, which builds up and dynamically manages personal profiles of consumers, aiming to facilitate and optimize the service discovery and recommendation process, in support of consumers' choices, thereby achieving the best quality of experience (QoE) as perceived by those consumers when utilizing different mobile services. The algorithm-driven recommended mobile services, accessible anytime-anywhere-anyhow through any kind of mobile devices via heterogeneous wireless access networks, range from typical telecommunication services (e.g., outgoing voice calls) to Internet services (e.g., multimedia streaming). These algorithms also may be further enriched by their being adapted and expanded to cover more sophisticated services such as helping the consumer's health and security needs, an example being the finding (with subsequent dynamic changing, if required) of the most 'healthy' or 'secure' driving/biking/jogging/walking route to follow so as to avoid areas posing particular, consumer-specific, health or safety risk.
\end{abstract}

Keywords: - Service recommendation, mobile services, smart system, real-time recommendations, Always Best Connected and best Served (ABC\&S), Wireless Billboard Channel (WBC).

Received: May 20, 2021. Revised: November 17, 2021. Accepted: December 1, 2021. Published: December 20, 2021.

\section{Introduction}

In the modern 'Big Data' era, recommendation systems have become the essential tools for both the providers and consumers for addressing the problem of information overload and assisting the latter in navigating through the vast 'information oceans' in order to find the information, product, item, or service needed. This process is highly dynamic with 
the increasing number of items, available for online buying, watching, etc., and the increasing number of mobile applications (apps) and services available. The goal of recommendation systems is to provide consumers with accurate timely recommendations [1]. So far, the efforts in this direction have been mostly focused on item recommendations, whereas app recommendations and service recommendations are left behind. The aim of this paper is to set out some new research contributions which begin to address the identified research gap in the use of recommendation systems, in particular for the benefit of mobile users (consumers). In doing this, the authors recognize they are pushing a new research and development (R\&D) horizon in the "service recommendation" sub-area, and one that could become quite extensive.

It's becoming ever clearer that consumers today seek more freedom of choice in choosing their desired (and 'best') mobile services. However, the increased freedom should not be a burden for the consumers themselves. Therefore, the provision of real-time service recommendations is a perceived demand to facilitate consumers with automatic discovery of the 'best' services (service instances) available for use through the 'best' mobile access network, following the consumer-oriented, consumer-friendly, and consumer-driven Always Best Connected and best Served (ABC\&S) paradigm. Such recommendations should cover a myriad of services already deployed, ranging from simple web browsing to sophisticated Internet of Things (IoT) services, by assisting consumers in discovering what they may need through the receipt of timely personalized suggestions and recommendations, or data in support of that to be used by an onboard recommendation app on their mobile device.

To this end, this paper presents a designed smart service recommendation system, hosted by a third party located in the cloud, which can automatically identify the usefulness and applicability of mobile services for each engaged consumer, and discover and recommend the 'best' instance of each service identified as useful for him/her. More specifically, the system seeks to dynamically provide consumers with an ordered list of ranked service instances addressing likely, and in a timely manner, specific consumer needs as may be interpolated or extrapolated from the present consumer behavior and profile. This thus supports the consumer at any moment choosing to use the 'best' instance of a particular service $\mathrm{s} / \mathrm{he}$ is interested in, matched to his/her needs. The recommendation of services is not only based on their relevance to a particular consumer (consumer context) but also takes into account the current constraints of the access network (network context) and the service context [2].

The main goal of the service recommendation system presented here is to provide consumers with a context-aware software solution that can assist them in choosing and selecting the 'best' service instances by means of a specially designed mobile app, which is installed on their mobile devices and works with the recommendation data supplied by third parties. This app facilitates and supports the consumer's service choice or may make this choice on behalf of the consumer when authorized by $\mathrm{him} / \mathrm{her}$ to do so. In both cases, the recommendation system would benefit greatly from the ability to make accurate predictions of consumers' preferences. This prediction may be the result of solving a combinatorial optimization problem, whereby each engaged consumer has specified criteria for making a particular service choice. For example, the mobile app besides having default 'pro-consumer' settings could of course facilitate consumer-defined configurations, e.g., the consumer may re-configure parameters such as the upper bound on the service cost, the lower bound on the quality of service (QoS), etc.

The difficulties in solving such an optimization problem are computational as the problem is NPcomplete itself, but also relate to the use of large and complex data structures. Thus, efficient heuristic methods must be used. An alternative approach for making predictions for service usage is to allow the recommendation system to discover patterns in the consumer behaviour, which must be continuously recorded, uploaded to, and stored in a distributed repository, supporting the operation of the corresponding recommendation system. Such repository can facilitate the effective intelligent mining of data on behalf of consumers, the outcomes of which may result in accurate and beneficial consumer behaviour predictions, and consumer service recommendations [3]. For the efficient data storage and offering of services in support of service recommendations based on the patterns discovered within the consumer data, the repository should be based 'in the cloud'. For this, notable cloud computing principles need to be employed along with effective 'big data' collection and processing techniques in order to facilitate the provision of service recommendations to consumers as an efficient solution, which is also commercially attractive to service providers. This form of cloud repository service may serve as a supplementary external support to the recommendation service 
provided internally by the mobile device having its own service recommendation app or as a fullservice recommendation system which a mobile user subscribes to, interacts with, and relies on. This paper addresses this latter solution.

Faced with the vast dynamically-changing information (consumer-, network-, and servicerelated), the elaborated smart recommendation system presents a scalable, efficient, and effective solution for real-time, cloud-based service recommendations, helped by 'big data' analytics [4]. This paper describes a refined structure of this system, based on the initial work, e.g., presented in [5], which is able to manage big sematic data in real-time for highly contextualized, customized, and personalized service recommendations. A Neo4j graph database is adopted for storing and managing the information about services and consumers in the form of a heterogeneous service network (HSN), in order to suggest most suitable and 'best' service instances. By exploiting context-aware recommendation approaches and semantic web technologies, the presented service recommendation system first collects and extracts service information, and dynamically models a HSN, based on the elaborated network schema. Then, profile kernels, referring to the minimal set of features describing the consumers' preferences, are extracted for modelling the consumers profiles. The discovery of consumers' features is performed, as proposed in [6], by utilizing the 'betweenness centrality' concept [7] within the scope of a HSN, which is envisaged as a novel approach for consumers profiles' generation based on the meta-paths concept.

The rest of the paper is organized as follows. The next section presents related work in the area of recommendations. Section 2 describes an application example of smart real-time recommendation of a mobile service instance. The design of the elaborated smart service recommendation system is presented in Section 4. Section 5 discusses the results achieved so far and future research directions. Finally, section 6 concludes the paper.

\section{Related Work}

The R\&D efforts in the main sub-areas of recommendations are presented briefly below, with a particular attention paid to service recommendations.

\subsection{Item Recommendations}

In this sub-area, a lot of work has been done, ranging from (e-)commerce - either by recommending items right at the spot, e.g., by means of digital menus for food recommendations at restaurants [8], or by recommending (substitute or complementary) items for online shopping at a retailer web site [9], or by recommending items for online shopping through a mediator, e.g., a video game [10] or a social media [11] (a.k.a. "social commerce" [12]) - to entertainment, e.g., recommendation of TV programs [13], streaming items (over social communities) [14], photos [15], music [16], etc., and even to e-learning, e.g., by recommending personalized online test questions for students [17]. In addition to these Business-toConsumer (B2C)-oriented and Consumer-toConsumer (C2C)-oriented directions, another much more complex one is the Business-to-Business (B2B) direction, e.g., [18] [19], due to its end-to-end (E2E), large-scale, and fuzziness attributes.

\subsection{App Recommendations}

Some work has been done also in the sub-area of app recommendations. For instance, [20] utilizes the consumer's location as a main context feature to recommend mobile apps that other consumers have installed/used frequently in the same location. [21] provides personalized app recommendations by combining item-based collaborative filtering (CF) with mobile apps' usage data related to the target consumer. In [22], a context-aware CF algorithm utilizing implicit consumer feedback is introduced, based on tensor factorization. [23] integrates the PMF principle (Probabilistic Matrix Factorization) [24] with the VEPM model (Version Evolution Progress Model) for providing app recommendations, based on the app version's description. In addition to the multiple academic research efforts, a wide range of commercial solutions have also appeared, such as Google Play ${ }^{1}$, Apple Store ${ }^{2}$, etc. Typically, these provide a list of similar apps when a consumer is browsing an app [25]. Usually this is done by app store optimization (ASO) techniques for optimizing the titles, descriptions, and keywords used in the apps as to make these more visible to potential consumers, e.g., by appearing in the recommendation sections of other apps, ranking high in the searching results, etc. However, the recommendation algorithms used are usually not made public [25]. Moreover, the recommendations provided in the current app market seem not very effective and not evenly distributed among the existing apps, either due to

\footnotetext{
${ }^{1} \mathrm{https}: / /$ play.google.com/store

${ }^{2}$ https://www.apple.com/store
} 
fraudulent app promotion (or manipulation of the recommendation system by some spamming app developers) or no-exposure behaviors, which may increase the exposure of some marginalized apps by making them more visible to potential consumers or constraining the exposure of other good apps to consumers. For instance, in the created recommendation network AppNet of Google Play, consisting of over 2 million apps and over 130 million app recommendation relations, the so-called "forgotten majority" (comprising 70\% of apps) seem to receive no recommendation links at all (even though some of these seem significantly popular, having millions of app installations!), while the "star" apps receive thousands of recommendations each [25].

\section{3 'Thing' Recommendations}

A relatively new direction in this regard relates to the 'thing recommendations' [26] within the IoT area, focusing on different application domains, such as intelligent transportation systems (ITS), smart city, smart home, smart health, smart environment control and protection, etc. Examples in the ITS area include computing and dynamically adjusting the optimal travel path(s) by a car navigation recommendation system, based on realtime traffic information [27], or generating and sending to drivers precise parking lot guiding suggestions by an urban car parking recommendation system [28] [29]. In the "smart health" area, examples of 'thing recommendations' relate to various personal-health reminders [28] with the goal of sending to the consumer up-to-date notifications, e.g., about lowest priced drugs available for sale in nearby pharmacies. In addition, this type of recommendations' approach can potentially support other "smart city" healthy-living applications, e.g., sending profile-based real-time alerts about areas of high/low pollen count, pollution, air quality index (AQI), etc., or more specific alerts relevant to a specific consumer as $\mathrm{s} /$ he moves around a city [28]. In the "smart home" area, recommendation examples include homes equipped with motion sensors monitoring the movements of elderly people living there and a supplementary system assisting them in their daily life activities, e.g., tracking (and reminding about) different steps needed to follow in order to complete a particular action such as taking their scheduled medicines or even as simple and routine as coffee/tea-making [26].

\subsection{Service Recommendations}

The sub-area of service recommendations has attracted great attention in the last few years. The work here ranges from recommending a specific aspect/parameter/feature/component of a single service to recommending a new instance of the same service or completely new service. A sophisticated example of the former are the adaptive video streaming recommendations - a form of in-app recommendations. By supporting various clientdriven bit-rate adaptation logic algorithms, this type of recommendation scheme could be used for optimally limiting the possible set of selectable bitrate levels and qualities of a video streaming service for the clients. Subsequently, each video streaming client uses the recommendations advanced as an upper bound together with its buffer level during the bit-rate selection process, which enables it to react quickly to sudden bandwidth variations and improve the quality of experience (QoE). By employing such recommendations for the optimal bit-rate level and quality, this method, called SDNDASH in the proposal set out in [30], avoids sub-optimal, purely client-driven network resource management and bitrate level decisions, whereby the available bandwidth is randomly distributed among the competing clients. Moreover, as a complete E2E solution (involving the server-, network-, and client side), SDNDASH can monitor and allocate network resources in a dynamic fashion as to maximize the QoE for each client, while at the same time avoiding the scalability issue when working with multiple clients competing for bandwidth in a shared network environment. The method also takes into consideration the consumer context in the form of mobile devices' capabilities, QoE, buffer occupancy, etc., to successfully tackle the problem of device heterogeneity (e.g., large-screen TVs vs. small-screen smartphones).

For the discovery and selection of IaaS cloud offerings, Zhang et al. [31] use a semi-automated, extensible, and ontology-based approach by utilizing a multi-criteria decision-making technique, based on real-time E2E QoS parameters for meeting the service-level agreement (SLA). By utilizing a mechanism of similarity computation and ranking of service providers based on consumer feedback, [32] proposes a service recommendation system, based on trust, reputation, and QoS requirements, for use in a Service Oriented Grid (SOG). For dynamic, runtime, proximity-based mobile service compositions, the authors in [33] utilize knowledgebased recommendation techniques. However, the smart service recommendation system, presented here, works as a global solution applicable to all 
types of mobile services, accessible via any type of mobile networks providing access to these and through any kind of mobile devices, anytimeanywhere-anyhow.

For service recommendations, the broker-based model is widely utilized [34], whereby an intermediary (broker) is used either to simply assist in the collaboration between consumers and service providers [35], or to match consumers and service providers through an auction mechanism [36], or to provide more comprehensive functionalities such as service packaging and monitoring for matching the requirements of consumers with the SLA offered by the service providers [37], and/or multi-attribute negotiation with service providers on behalf of the consumer and monitoring the behaviors of the providers to help consumers fulfill their functional and QoS requirements [38]. All this could be done either with respect to a single service or a (credible) composite service [39] [40]. By providing such functionalities and intermediate services between consumers and service providers, brokers of course can make profit [34]. To make the right decisions, the brokers use either consumer behavior [41] and/or historical records of consumers [42] and/or direct consumer experience and feedback [43], or calculate consumers' aggregated requirements (e.g., for service cost, execution time, reliability, availability, responsiveness, etc.) to find an optimized match of these with available service offerings at any time or place for the consumer [44], or use machine learning techniques to better understand consumers' preferences, even in the presence of implicit consumer feedback [34]. Of course, it would be better to use a combination of these methods while also considering the security and trust assurance in service matching [45] [46] [47] as to eliminate spurious services or consider service performance deterioration, e.g., as a result of malicious attacks. Furthermore, the broker could use either a centralized [43] or distributed model [34], with the latter allowing for the existence of multiple brokers acting even as competitors to each other. Similar categorization applies to the utilized trust management schemes - these could be either centralized [43] or distributed [34]. Adding a (self) learning ability to the brokers, as in [34], is important for allowing them initially to operate in unfamiliar environments, gradually increase their competence, understand better consumers' preferences, and optimize their own behaviors even in the case of implicit consumer feedback. In any case, consumers' freedom, to accept or not the service recommendations supplied by the broker(s) should not only always be respected but facilitated (e.g., by ever better GUIs, consumer-interactive option setting, etc.). Such a principle is not only in harmony with most national legislative frameworks supporting consumer autonomy and privacy, but also with the principle of the "consumer being the driver' of services and service-usage.

The challenge of getting dynamic updates of any available service offerings, which are location- and time-dependent, to the brokers, and updating their responses sent back as recommendations to the consumers in a reliable timely manner, is a significant technical one not dealt with reasonably in those otherwise excellent proposals in [31]-[37]. Nor are the significant scaling problems addressed of background communications activity this kind of recommendations service infrastructure will generate and the computation resources, whether centralized or distributed, which will be required.

A simpler, scalable, more feasible alternative solution is elaborated in [48] [49], whereby all services' offerings, with all their defining details, may be conveyed to the consumer devices through normally-on, live, latent, simplex, background services' advertisement channels such as Wireless Billboard Channels (WBCs). In this model all the recommendation algorithmic schemes would reside on each consumer device, absorb this information, and compile recommendations 'in situ.' Such a scheme may be referred to as an independent, autonomous mobile service recommendation app. Brokers could and would still have an important role here both in mediating between the service providers and the business of managing the WBC advertisements and channels, as well as the provision and updating of recommendation system algorithms on consumer devices. All of such supports have attractive business solutions, as indicated in [48] [49]. Also, as pointed out there, a certain amount of global standardization in relation to protocol interfaces and data structures would help enormously, enabling both consumers to access relevant mobile services from multiple service providers, and those service providers having ready access to all consumers, all of which benefit from service offering commercial competition. This kind of mobile-based recommendation system/app is an important aspect of the service popularization task performed by the smart recommendation systems related to the kind presented in this paper but is not dealt with further here in any great depth for reason of space, apart from a mention of it being included in Section 4 in elaboration of the recommendation system architecture presented in Fig. 3.

The smart service recommendation system, presented here, follows the centralized brokerage 
type, by also adding a trust factor. Nonetheless, much about the operational concepts of the scheme, presented here, may be transferable to the independent, autonomous mobile service recommendation technology.

\section{Application Example}

The smart service recommendation system, presented here, can provide each engaged consumer with a contextualized, customized, and personalized list of 'best' instances of the preferred mobile services by considering the current consumer-, network-, and service context. An application example in Fig. 1, based on an outgoing voice call service, briefly illustrates the idea [5]. In this example, consumer $A$ wants to call consumer $B$ always in the 'best' possible way, e.g., based on the integral 'cost/quality' criterion. The cheapest option, presented in green color in Fig. 1, would be to utilize the VoIP technology, e.g., by using the mobile app, provided by some VoIP provider, to initiate an outgoing call over a (free or paid) $\mathrm{Wi}-\mathrm{Fi}$ connection. This Wi-Fi/VoIP option will be the preferable one when consumer $B$ is a friend or a family member, as this involves a minimum cost even though the quality guarantees and supports will be the lowest. However, in doing this, consumer $A$ could miss an opportunity of using another/better VoIP service provider, who supplies better rates for making a voice call to that particular consumer $B$ at that moment. The elaborated smart service recommendation system, presented in this paper, can find such an opportunity, i.e., the existence of a better VoIP service instance, and recommend it in real time to consumer $A$.

The second option, presented in red color in Fig. 1 , would be to use cellular (3G-5G) connection. This high-quality, higher-cost option will be the preferable one in certain contexts such as when consumer $B$ is the $A$ 's manager, business partner, important client, etc. The situation, however, may change drastically when one (or both) of the involved consumers is currently in a foreign country due to the associated added roaming cost, which can typically double the total call cost. In this case, additional and more attractive option, presented in orange color in Fig. 1, is to use a local SIM card which is on sale in the corresponding foreign country, instead of the SIM card provided by the home country's cellular operator. This is the preferable option for making phone calls abroad, especially in countries where the home operator was unable to negotiate better/lower roaming costs for its subscribers. Yet, the best option, if a high-quality voice call is required, would be the use of a global
SIM card, which in many cases could deliver the lowest cost. The use of another, not-home SIM card, even in case when it is an eSIM card, comes with difficulties in finding if that particular card indeed provides competitive cost for calling that particular consumer $(B)$, as it depends also on his/her current location (the country where $\mathrm{s} / \mathrm{he}$ is at the moment of the call).

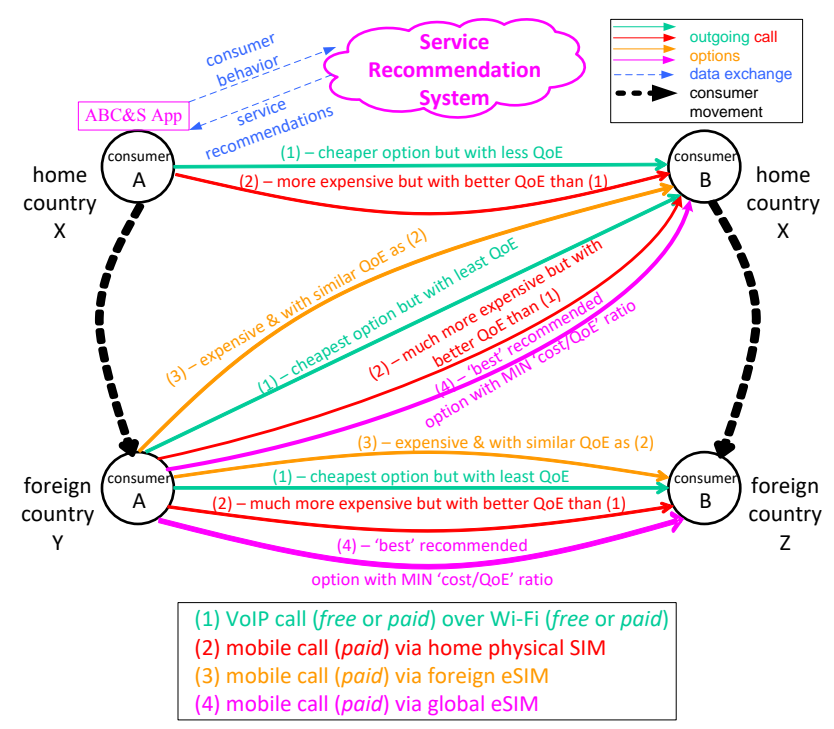

Fig. 1. An application example of smart real-time recommendation of an outgoing voice call service instance.

All these scenarios can be easily served by the smart service recommendation system, presented in the next section, which can find the 'best' option for making a call by consumer $A$ to consumer $B$ in each particular case, depending on the current context. For this, the system works in cooperation with an $A B C \& S$ app, operating on the mobile device of each engaged consumer, e.g., for recommending to consumer $A$ the 'best' outgoing voice call option in the example considered here. Moreover, if the consumer profile settings are so set, the app could be used also to automatically select the 'best' service instance, depending on the current context.

\section{Smart Service Recommendation System}

The elaborated smart system supplies service recommendations in two directions: (i) service popularization, by providing a global ranked list of popular services to all engaged consumers; and (ii) service personalization, by providing a personalized ranked list of top- $N$ service instances to a particular consumer. For the latter, for each consumer, a recommendation engine first calculates the 
prediction score for each unrated service instance and then recommends the top- $N$ service instances with the highest scores to that consumer.

A hybrid context-aware recommendation approach, utilizing both pre-filtering and postfiltering phases, and considering different contextual information (of the consumer, network, and service), was elaborated, initially in [50]. As each recommendation model has its own pros and cons, many researchers have suggested to combine multiple models in a variety of manners [51]. Subsequently, a complex hybridization was elaborated for the presented smart service recommendation system, which according to the categorization provided in [51], could be labeled as cascade, weighted, and mixed at the same time. Cascade hybridization works in stages, whereby one recommendation technique refines the recommendations given by another in the previous stage. In mixed hybridization [51], recommendations from several different recommenders are presented at the same time. The service recommendation system, presented here, utilizes mixed hybridization followed by weighting in order to come up with the final ranking of services. With weighted hybridization, the scores of several recommendation models are combined in a single formula with weighted coefficients to produce a single recommendation.

A more developed version of the smart service recommendation system, initially proposed in [5], is presented here below, for finding and recommending to consumers the 'best' instances of mobile services. Each mobile service is described by a respective service description (SD), which is quite complex for some service types.

Deciding on which service instance is the 'best' one in each case is based on a set of context parameter values, categorized in three groups consumer-related $(\boldsymbol{c})$, service-related $(\boldsymbol{s})$, and (access) network-related $(\boldsymbol{n})$, forming a 3D $(\boldsymbol{c}, \boldsymbol{s}, \boldsymbol{n})$ context space, as illustrated in Fig. 2. This way, the elaborated service recommendation system supports real context-awareness, by utilizing a diverse range of (explicit and implicit) data available in a rapid and scalable manner [22]. Service recommendations depend greatly on the current context as being the key element in the mobile space along with the proactive nature of mobile services (and their recommendations) needed for facilitating the consumer input and effective interaction with services. In addition, taking the context into account has really a substantial impact on the recommendation performance [22]. Moreover, the concept of context allows making smart decisions based on mining of data, stored in cloud repositories. In [2], it was proposed for the context to include both the data sensed in the environment (as in a typical context-aware system), the behavior history of the consumer, and the collective behavior history of consumers who have acted in a similar environment.

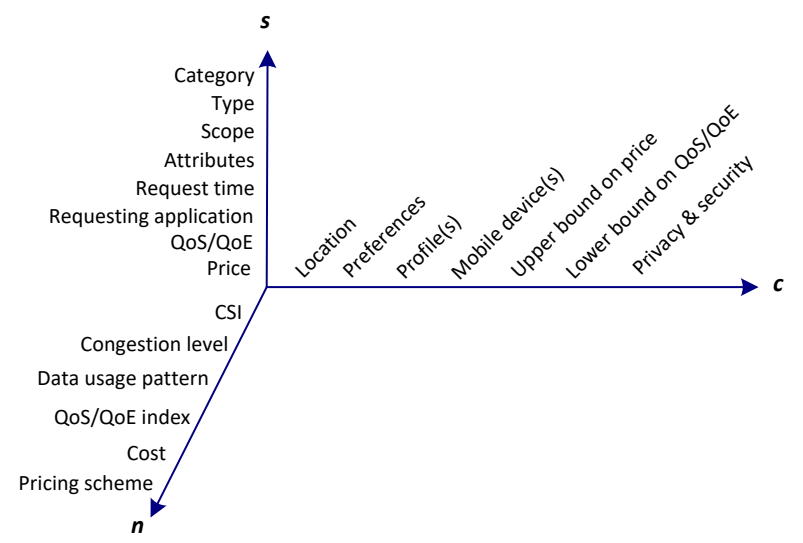

Fig. 2. The 3D context space.

The developed smart service recommendation system continuously updates the consumer behavior profiles, consumer interests and requirement tendencies, and sends a corresponding up-to-date ranked list of 'best' recommended service instances to each engaged consumer, by utilizing relevant recommendation algorithms [52] [53] and updated recommendation rules. A modular system structure has been adopted in [5] with three domains, related to the consumer, web, and cloud, respectively (Fig. $3)$. More details on these domains are provided in the following subsections.

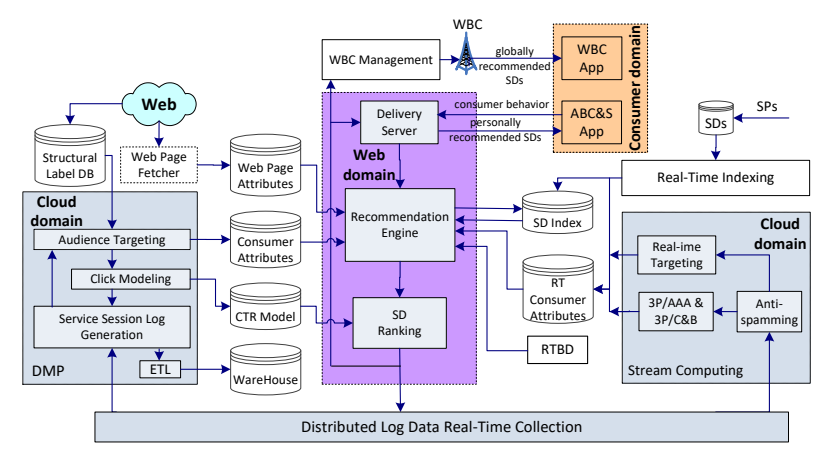

Fig. 3. The modular structure of the cloud-based smart service recommendation system. 


\subsection{Consumer Domain}

In this domain, an ABC\&S app [54] operates on the mobile device of the engaged consumer as to visualize to him/her the 'best' service instances recommended by the system, but also to facilitate the consumer-driven (or service-provider-initiated) handover from one access network to another ('best') access network, if needed. If the consumer profile is set accordingly, the app could also automatically select the 'best' instance of each mobile service of interest to the consumer, depending on the current context. The app design was elaborated initially in [55] [5].

In addition, for the 'service popularization' option, a WBC app runs on the consumer's mobile device for receiving and processing the list of new/popular/emerging services, created by a corresponding WBC management module of the system, whose SDs are cached by it for broadcasting (on WBCs) and pushing to the mobile devices. The design and development of a three-layer software architecture for this app have been described in detail in [56]. It is through here also that the independent, autonomous mobile service recommendation technology and app, when present, will source much of its data on geo-dependent mobile services available, which would be fed through the WBCs, being made up of a mixture of relevant data flows from free and/or subscribed services typically provided by the cloud-based mobile service recommendation systems and such like providers.

\subsection{Web Domain}

One of the key parts in this domain is the delivery server, which receives and aggregates information about consumer behavior (e.g., the searches, tags, reviews, etc., for services and associations with services done by the corresponding consumer, the actual service usage, etc.), collected by the ABC\&S app and other third parties involved in the process (e.g., social media platforms), and sends back (to the ABC\&S app) recommendations about the 'best' instance of each mobile service of interest to the corresponding consumer. Acting as a central part in the web domain, the recommendation engine facilitates uploading of recommendation algorithms to the system, and defining and updating of recommendation rules. For supporting real-time recommendations and off-time analytical operations, the engine was built with a Lambda architecture [57]. Through its SD retrieval module, the engine can search the SD index database based on relevant keywords. Facilitated by three databases (i.e., RTBD, consumer attributes, and web page attributes), the recommendation engine produces an initial service recommendation list, which is sent to the SD ranking module. After relevant processing done by the CTR module, a final personalized list of top- $N$ recommended service instances is generated, which is sent to the $\mathrm{ABC} \& \mathrm{~S}$ app (via the delivery server) and simultaneously pushed to the distributed log data real-time collection module. In addition, a globally recommended list of services is generated by the SD ranking module for a global delivery to consumers via the WBC infrastructure.

\subsection{Cloud Domain}

Here the consumer data, harvested via mobile devices and collected by other means, is stored and mined. Based on the analysis of this data, the cloud is able to predict the applicability (and ABC\&S suitability) of mobile services to each engaged consumer. Over long periods of time, the collected data can provide an accurate view of particular consumer cohorts, based on their common interests, repetitive access to and usage of particular services, etc. By monitoring this information, the service recommendation system can accurately predict the types and instances of services most applicable and suitable to individuals, and in turn, recommend the 'best' instances of these to consumers.

The cloud domain consists of three main parts, described in the following subsections.

\subsubsection{Data Management Platform (DMP)}

Acting as a machine learning platform, the DMP [58] [59] is able to transform the collected raw data of various types into actionable analytic dataset, i.e., consumer behavior profiles. These include consumers' preferences for services and service content, consumption preferences, shopping preferences, interest preferences, app usage, etc., abiding by the consumer-privacy principles. TABLE I. lists the main features included in the consumer profiles, [57].

In general, DMP is used to generate audience segments, i.e., to recommend to engaged consumers the 'best' mobile service instances. More specifically, DMP supports the corresponding data collection, processing, analytics, and consumer targeting operations. For this, it utilizes real-time consumer's profiling algorithms and off-time data mining algorithms [57], and has been implemented with a Publish/Subscribe design pattern [59].

The corresponding service session data is created with a unique consumer ID serialized to a data warehouse by the ETL module. Then the audience targeting module collects the data and updates it to the consumer attributes key-value database and to 
the CTR model database. Besides collecting the log data, DMP also fetches the corresponding service's web page, updates the web page attributes database, labels the keywords, and saves the new data into a structural label database.

TABLE I. MAIN FEATURES IN CONSUMER PROFILES.

\begin{tabular}{|c|c|}
\hline Profiles & Features \\
\hline $\begin{array}{l}\text { Internet } \\
\text { Features }\end{array}$ & $\begin{array}{l}\text { Statistics of time spent online; make } \\
\text { and model of mobile device; operating } \\
\text { system; browser type. }\end{array}$ \\
\hline $\begin{array}{l}\text { Marketing } \\
\text { Features }\end{array}$ & $\begin{array}{l}\text { Consumption cycle; consumption } \\
\text { amount; industry-related info; } \\
\text { marketing-related info; price-related } \\
\text { info. }\end{array}$ \\
\hline $\begin{array}{l}\text { Demographic } \\
\text { Features }\end{array}$ & $\begin{array}{l}\text { Gender, age, location, price acceptance } \\
\text { level; marriage status; lifestyle; body } \\
\text { shape; work type. }\end{array}$ \\
\hline $\begin{array}{l}\text { Consumer } \\
\text { Reviews } \\
\text { Features }\end{array}$ & $\begin{array}{l}\text { Service reviews, ratings, comments, } \\
\text { tags, etc.; creation time; consumer ID; } \\
\text { additional info. }\end{array}$ \\
\hline $\begin{array}{l}\text { Action History } \\
\text { Features }\end{array}$ & $\begin{array}{l}\text { Service ID; time stamp; consumer's } \\
\text { action; other related action history. }\end{array}$ \\
\hline $\begin{array}{l}\text { Category } \\
\text { Features }\end{array}$ & $\begin{array}{l}\text { Category ID; } \quad \text { score; } \\
\text { preferences; creation time. }\end{array}$ \\
\hline
\end{tabular}

\subsubsection{Stream Computing}

This part is a real-time computing subsystem, which uses an anti-spam functionality to filter the consumer behavior, integrates a (third-party) authentication, authorization, and accounting (3P/AAA) and (third-party) charging and billing (3P/C\&B) functionality for services, and updates the behavior to the real-time consumer attributes Redis database.

\subsubsection{Distributed Log Data Real-Time Collection} This part utilizes a producer-consumer paradigm for supporting the data exchange between the web domain (acting as a producer) and the cloud domain (acting as a consumer). A Flume-based distributed message structure has been elaborated for collecting all $\log$ data required and serializing these in the cloud.

\section{Discussion}

The smart service recommendation system, presented in this paper, so far utilizes only learning- based recommendation techniques, mainly utilizing the CF approach, e.g., [52] [53]. However, these techniques work best for dedicated consumers willing to spent some time in making their preferences known to the system [51]. In order to include also the casual consumers, the ramp-up problem must be tackled. In this regard, utilitybased [60] and knowledge-based [61] recommendation approaches should be explored in the future as these do not rely on having the consumers preferences' history at their disposal. In addition, their recommendations are based solely on the evaluation of the match that exists between consumers' needs and service instance offerings currently available.

The utility-based approach seems more attractive due to its ability to factor non-product attributes, such as service providers' reliability and/or services' availability, security, responsiveness, etc., into a utility function, allowing consumers to express a wide range of matters to be considered for service recommendations. For consumers having some flexibility, this allows a tradeoff of service price against service delivery schedule, for instance. Another advantage of this approach relates to its sensitiveness to any changes in consumers' preferences. However, its recommendation ability is static, resulting in providing service recommendations to a particular consumer only based on the computation of the utility of each service instance. This means that if this approach is to be followed, the smart service recommendation system, presented here, must build a complete utility function across all features of all service instances, which is a time-consuming process which should be continuously performed with each new service instance (dis)appearing. In addition, constraint satisfaction techniques must be employed by the system as to locate the best match, and consumer profiles will be consumer-specific utility functions.

The knowledge-based recommendation approach suggests objects based on inference about the consumers' needs and their preferences. For this, it seeks to algorithmically intuit about relationships between the consumers' needs and possible recommendations of service instances. The knowledge forms are of three types: (1) catalog knowledge, e.g., about the service instances being recommended and their features; (2) functional knowledge, i.e., how a particular service instance meets the needs of a particular consumer; and (3) consumer knowledge, e.g., in the form of a general demographic or specific information about the consumer. In this case, the consumer profiles will include any knowledge that supports this inference. 
Compared to the utility-based recommendation approach, the additional advantage here relates to the achievable mapping of consumers' needs to service instances. Moreover, the knowledge-based recommendation approach seems appropriate for casual explorations because it demands less from the consumers than the utility-based approach. A knowledge engineering, however, is required [51].

A possible combining of these two recommendation approaches for utilization by the presented smart service recommendation system seems to be a good choice for future exploration as the calculation of utility value for recommended service instances could be well-based on functional knowledge [51]. Moreover, both approaches do not suffer from ramp-up and sparsity problems since recommendations are not grounded on accumulated statistical evidence. In addition, both approaches respond to the immediate needs of consumers and do not require any retraining when consumers' preferences change [51]. This is achievable either by requesting consumers to perform their own mapping of needs to service features, e.g., in the form of preference functions for each feature or by answering interactive, detailed questionnaires [51], or otherwise by inferencing this from the consumers' interaction with service instances, which is envisaged as more appropriate tactic for utilization by the elaborated system. However, in order to avoid the burden of interaction associated with constructing a complete preference function by a consumer, along with weighting the significance of each possible service feature, a small number of 'stereotype' preference functions should be provided, as suggested in [51], as to get the consumer started. Of course, s/he needs to look at the weigh and select a preference function for each feature of a service of interest, but this seems feasible as services usually have only a few features, e.g., cost, QoS/QoE, availability, security, responsiveness, etc.

\section{Conclusion}

Smartphones are currently the most popular consumer electronic devices, creating a new media platform with their anytime-anywhere accessible functionalities. However, the most crucial problem for service providers is related to the delivery of relevant mobile services to the right consumers, by taking into account the current context. The developed smart service recommendation system, presented in this paper, can assist mobile service providers to reach valuable consumers, by supplying to them an up-to-date list of ranked service instances to choose from. By using the designed system, consumers can receive timely recommendations about the 'best', for them, instances of mobile services, at any moment and in any location, and in addition to use these via the 'best' available mobile access network, thus realizing a truly consumerdriven always best connected and best served $(\mathrm{ABC} \& S)$ paradigm realization.

The presented smart service recommendation system facilitates the storage of data harvested from consumers' mobile devices and third parties, such as social media platforms, and based on the 'big data' analytics offers predictions as to applicability (and the ABC\&S suitability) of services to each engaged consumer. Over time, the collected filtered data can produce an accurate view of particular consumer cohorts, based on their common interests, repetitive access to and usage of particular services, etc. By using this information, the service recommendation system is able to accurately predict the types of services most applicable to a particular consumer, and in turn, recommend these to him/her in realtime manner. Furthermore, efficient artificial intelligence algorithms will be implemented into the system so as to facilitate better service utilization predictions in the cloud. The collaboration option of this cloud with wireless billboard channels' (WBC) [48] [49] service providers holds potential. Through it, consumers may have their information distilled by the cloud, relevant to their current context, delivered to them in a personalized frame through an appropriate WBC. The schema for this has been suggested in outline, but full elaboration of this interesting approach first needs further research.

\section{Acknowledgement}

The authors would like to acknowledge the invaluable technical support and advice in regard to this paper and the underpinning research work which they received from Dr. M. O'Droma, University of Limerick, Ireland.

\section{References:}

[1] Jalili, M., et al., Evaluating Collaborative Filtering Recommender Algorithms: A Survey. IEEE Access, 2018. 6: p. 7400374024.

[2] Ganchev, I., M. O'Droma, N. Nikolov, Z. Ji, A UCWW Cloud System for Increased Service Contextualization in Future Wireless Networks, in 2nd International Conference on Telecommunications and Remote Sensing (ICTRS'13). 2013: Noordwijkerhout, The Netherlands. p. 69-78.

[3] Witten, I., E. Frank, and M. Hall, Data Mining: Practical Machine Learning Tools 
and Techniques. 3rd ed. 2011: Morgan Kaufmann. 664.

[4] Zhang, H., et al., A Service Recommendation System for UCWW, in 4th UL-NUIG Alliance Postgraduate Research Day 2014. 2014: Limerick, Ireland. p. x-1.

[5] Ganchev, I., et al., Smart recommendation of mobile services to consumers. IEEE Transactions on Consumer Electronics, 2017. 63(4): p. 499-508.

[6] Zhang, H., et al. A service recommendation model for the Ubiquitous Consumer Wireless World. in 2016 IEEE 8th International Conference on Intelligent Systems (IS). 2016.

[7] Barthélemy, M., Betweenness centrality in large complex networks. The European Physical Journal B - Condensed Matter and Complex Systems, 2004. 38: p. 163-168.

[8] Gunawardena, D. and K. Sarathchandra. BestDish: A Digital Menu and Food Item Recommendation System for Restaurants in the Hotel Sector. in 2020 International Conference on Image Processing and Robotics (ICIP). 2020.

[9] Ahsan, U., et al. Complementary Recommendations Using Deep Multi-modal Embeddings For Online Retail. in 2020 IEEE International Conference on Big Data (Big Data). 2020.

[10] Bertens, P., et al. A Machine-Learning Item Recommendation System for Video Games. in 2018 IEEE Conference on Computational Intelligence and Games (CIG). 2018.

[11] Gao, C., et al., Cross-platform Item Recommendation for Online Social ECommerce. IEEE Transactions on Knowledge and Data Engineering, 2021: p. 1-1.

[12] Gao, C., et al., Item Recommendation for Word-of-Mouth Scenario in Social ECommerce. IEEE Transactions on Knowledge and Data Engineering, 2020: p. 1-1.

[13] Lee, S., D. Lee, and S. Lee, Personalized DTV program recommendation system under a cloud computing environment. IEEE Transactions on Consumer Electronics, 2010. 56(2): p. 1034-1042.

[14] Zhou, X., et al. Online Social Media Recommendation Over Streams. in 2019 IEEE 35th International Conference on Data Engineering (ICDE). 2019.

[15] Tian, Y., et al., An enhanced personal photo recommendation system by fusing contextual and textual features on mobile device. IEEE Transactions on Consumer Electronics, 2013. 59(1): p. 220-228.
[16] Rosa, R.L., D.Z. Rodriguez, and G. Bressan, Music recommendation system based on user's sentiments extracted from social networks. IEEE Transactions on Consumer Electronics, 2015. 61(3): p. 359-367.

[17] Zhou, Y., et al. An estimating algorithm for English levels of student and difficulties of test question. in Proceedings of 2013 3rd International Conference on Computer Science and Network Technology. 2013.

[18] Nia, A.G., et al. A Framework for a LargeScale B2B Recommender System. in 2019 IEEE 14th International Conference on Intelligent Systems and Knowledge Engineering (ISKE). 2019.

[19] Wu, D., G. Zhang, and J. Lu, A Fuzzy Preference Tree-Based Recommender System for Personalized Business-to-Business EServices. IEEE Transactions on Fuzzy Systems, 2015. 23(1): p. 29-43.

[20] Woerndl, W., C. Schueller, and R. Wojtech. A Hybrid Recommender System for Contextaware Recommendations of Mobile Applications. in 2007 IEEE 23rd International Conference on Data Engineering Workshop. 2007.

[21] Yan, B. and G. Chen, AppJoy: personalized mobile application discovery, in Proceedings of the 9th international conference on Mobile systems, applications, and services - MobiSys '11. 2011, Association for Computing Machinery: Bethesda, Maryland, USA. p. 113-126.

[22] Karatzoglou, A., et al., Climbing the app wall: enabling mobile app discovery through context-aware recommendations, in Proceedings of the 21st ACM international conference on Information and knowledge management - CIKM '12. 2012, Association for Computing Machinery: Maui, Hawaii, USA. p. 2527-2530.

[23] Maheswari, M., et al., PEVRM: Probabilistic Evolution Based Version Recommendation Model for Mobile Applications. IEEE Access, 2021. 9: p. 20819-20827.

[24] Salakhutdinov, R. and A. Mnih, Probabilistic Matrix Factorization, in Proceedings of the 20th International Conference on Neural Information Processing Systems. 2007, Curran Associates Inc.: Vancouver, British Columbia, Canada. p. 1257-1264.

[25] Guo, Q., et al., AppNet: understanding app recommendation in Google Play, in Proceedings of the 3rd ACM SIGSOFT International Workshop on App Market 
Analytics - WAMA 2019. 2019, Association for Computing Machinery: Tallinn, Estonia. p. 19-25.

[26] Altulyan, M., et al., A Survey on Recommender Systems for Internet of Things: Techniques, Applications and Future Directions. The Computer Journal, 2021.

[27] Songhui, L., et al. On key technology of service recommendation system for car navigation. in Proceedings of the 31 st Chinese Control Conference. 2012.

[28] Zhang, H., et al., A Hybrid Service Recommendation Prototype Adapted for the UCWW: A Smart-City Orientation. Wireless Communications and Mobile Computing, 2017. 2017: p. 1-11.

[29] Wu, E.H., et al., Agile Urban Parking Recommendation Service for Intelligent Vehicular Guiding System. IEEE Intelligent Transportation Systems Magazine, 2014. 6(1): p. 35-49.

[30] Bentaleb, A., A.C. Begen, and R. Zimmermann, SDNDASH: Improving QoE of HTTP Adaptive Streaming Using Software Defined Networking, in Proceedings of the 24th ACM international conference on Multimedia. 2016, Association for Computing Machinery: Amsterdam, The Netherlands. p. 1296-1305.

[31] Zhang, M., et al., An Infrastructure Service Recommendation System for Cloud Applications with Real-time QoS Requirement Constraints. IEEE Systems Journal, 2017. 11(4): p. 2960-2970.

[32] Nagarathna, N., M. Indiramma, and J.S. Nayak. Optimal Service Selection Using Trust Based Recommendation System for ServiceOriented Grid. in 2012 International Symposium on Cloud and Services Computing. 2012.

[33] Pääkkö, J., et al. Applying Recommendation Systems for Composing Dynamic Services for Mobile Devices. in 2012 19th Asia-Pacific Software Engineering Conference. 2012.

[34] Li, W., et al., TSLAM: A Trust-enabled SelfLearning Agent Model for Service Matching in the Cloud Market. ACM Transactions on Autonomous and Adaptive Systems, 2019. 13(4): p. 1-41.

[35] Buyya, R., C.S. Yeo, and S. Venugopal. Market-Oriented Cloud Computing: Vision, Hype, and Reality for Delivering IT Services as Computing Utilities. in 2008 10th IEEE International Conference on High
Performance Computing and

Communications. 2008.

[36] Song, B., M.M. Hassan, and E. Huh. A Novel Cloud Market Infrastructure for Trading Service. in 2009 International Conference on Computational Science and Its Applications. 2009.

[37] Wagle, S.S. SLA Assured Brokering (SAB) and CSP Certification in Cloud Computing. in 2014 IEEE/ACM 7th International Conference on Utility and Cloud Computing. 2014.

[38] Badidi, E. A Cloud Service Broker for SLAbased SaaS provisioning. in International Conference on Information Society (i-Society 2013). 2013.

[39] Chun-hua HU, Ji-bo LIU, and J.-x. LIU, Services selection based on trust evolution and union for cloud computing. Journal on Communications, 2011.32(7): p. 71-79.

[40] Arifulina, S., et al. Market-Specific Service Compositions: Specification and Matching. in 2015 IEEE World Congress on Services. 2015.

[41] Deng, T. Analysis of user behavior in cloud broker. in 2017 8th IEEE International Conference on Software Engineering and Service Science (ICSESS). 2017.

[42] Aazam, M. and E. Huh. Broker as a Service (BaaS) Pricing and Resource Estimation Model. in 2014 IEEE 6th International Conference on Cloud Computing Technology and Science. 2014.

[43] Li, X., et al., T-Broker: A Trust-Aware Service Brokering Scheme for Multiple Cloud Collaborative Services. IEEE Transactions on Information Forensics and Security, 2015. 10(7): p. 1402-1415.

[44] Rane, D. and A. Srivastava. Cloud Brokering Architecture for Dynamic Placement of Virtual Machines. in 2015 IEEE 8th International Conference on Cloud Computing. 2015.

[45] Wang, Y., J.-t. Zhou, and H.-y. Tan, $C C$ PSM: A Preference-Aware Selection Model for Cloud Service Based on Consumer Community. Mathematical Problems in Engineering, 2015. 2015: p. 1-13.

[46] X. Meng, et al., Trust and behavioral modeling based two layer service selection. J. Xidian Univ., 2014. 41(4): p. 198-204.

[47] Li, X., J. He, and Y. Du, Trust Based Service Optimization Selection for Cloud Computing. International Journal of Multimedia and 
Ubiquitous Engineering, 2015. 10(5): p. 221230.

[48] Flynn, P., I. Ganchev, and M. O'Droma. $W B C s$ - ADA vehicle and infrastructural support in a UCWW. in 2006 IEEE Tenth International Symposium on Consumer Electronics (ISCE'06). 2006. St. Petersburg, Russian Federation.

[49] Ji, Z., I. Ganchev, and M. O'Droma, Advertisement Data Management and Application Design in WBCs. Journal of Software, 2011. 6(6): p. 1001-1008.

[50] Zhang, H., N.S. Nikolov, and I. Ganchev. UCWW semantic-based service recommendation framework. in 2015 IEEE International Symposium on Technology and Society (ISTAS). 2015.

[51] Burke, R., Hybrid Recommender Systems: Survey and Experiments. User Modeling and User-Adapted Interaction, 2002. 12(4): p. 331-370.

[52] Zhang, H., et al., FeatureMF: An Item Feature Enriched Matrix Factorization Model for Item Recommendation. IEEE Access, 2021. 9: p. 65266-65276.

[53] Zhou, D., et al., Novel SDDM Rating Prediction Models for Recommendation Systems. IEEE Access, 2021. 9: p. 101197101206.

[54] Ganchev, I., Z. Ji, and M. O'Droma. UCWW Cloud-based ABC\&S Mobile App. in 1st URSI Atlantic Radio Science Conference (URSI AT-RASC 2015). 2015. Gran Canaria, Canary Islands.: IEEE.

[55] Ganchev, I., Z. Ji, and M. O'Droma, A cloudbased service recommendation system for use in $U C W W$, in 11th International Symposium on Wireless Communication Systems (ISWCS'2014). 2014, IEEE: Barcelona, Spain. p. 791-795.

[56] Ji, Z., I. Ganchev, and M. O'Droma, An iWBC consumer application for 'always best connected and best served': design and implementation. IEEE Transactions on Consumer Electronics, 2011. 57(2): p. 462470 .
[57] Ganchev, I., Z. Ji, and M.O. Droma. A conceptual framework for building a mobile services' recommendation engine. in 2016 IEEE 8th International Conference on Intelligent Systems (IS). 2016.

[58] Ganchev, I., Z. Ji, and M.O. Droma. A data management platform for recommending services to consumers in the UCWW. in 2016 IEEE International Conference on Consumer Electronics (ICCE). 2016.

[59] Ganchev, I., Z. Ji, and M.O. Droma. The creation of a data management platform for use in the UCWW. in 2016 SAI Computing Conference (SAI). 2016.

[60] Zheng, Y. and A. Pu. Utility-Based MultiStakeholder Recommendations by MultiObjective Optimization. in 2018 IEEE/WIC/ACM International Conference on Web Intelligence (WI). 2018.

[61] Gyrard, A. and A. Sheth, IAMHAPPY: Towards an IoT knowledge-based crossdomain well-being recommendation system for everyday happiness. Smart Health, 2020. 15: p. 100083.

Ivan Ganchev carried out the conceptualization, methodology, project administration and supervision, funding acquisition, writing of the original draft and editing of the final paper.

Zhanlin Ji carried out data curation, formal analysis, investigation, validation, software development, and resource management.

This publication has emanated from joint research conducted with the financial support of the Bulgarian National Science Fund (BNSF) under the Grant No. KP-06-IP-CHINA/1 (КП06-ИП-КИТАЙ/1) and the S\&Т Major Project of the Science and Technology Ministry of China, Grant No. 2017YFE0135700.

\section{Creative Commons Attribution License 4.0 (Attribution 4.0 International, CC BY 4.0)}

This article is published under the terms of the Creative Commons Attribution License 4.0 https://creativecommons.org/licenses/by/4.0/deed.en US 